MATHEMATICS OF COMPUTATION

Volume 72 , Number 241 , Pages 375-385

S $0025-5718(02) 01444-8$

Article electronically published on June 25, 2002

\title{
CONVERGENCE OF THE UNITARY $Q R$ ALGORITHM WITH A UNIMODULAR WILKINSON SHIFT
}

\author{
TAI-LIN WANG AND WILLIAM B. GRAGG
}

\begin{abstract}
In applying the $Q R$ algorithm to compute the eigenvalues of a unitary Hessenberg matrix, a projected Wilkinson shift of unit modulus is proposed and proved to give global convergence with (at least) a quadratic asymptotic rate for the $Q R$ iteration. Experimental testing demonstrates that the unimodular shift produces more efficient numerical convergence.
\end{abstract}

\section{INTRODUCTION}

To compute the eigenvalues of a unitary Hessenberg matrix, nonzero shifts must be used in applying the $Q R$ algorithm to avoid cycling and invariance of the matrix [5]. In a previous work 9] we showed that global convergence of the $Q R$ iteration is guaranteed with the Wilkinson shift in the unitary case, if only the starting value of the shift sequence is not zero, and an initial-value modification of the shift was proposed in the exceptional case (when the starting shift is zero). A general mixed shift strategy, devised with selective use of the modified Rayleigh and Wilkinson shifts, was also presented. The asymptotic rate of convergence was shown to be cubic with these shift strategies. Nevertheless, there are special unitary Hessenberg matrices in which decrease of the last subdiagonal element can be extremely slow in the early stages of the $Q R$ iteration. As numerical examples illustrate (see Section 4 ), it may take a considerable number of iterations in such special cases for the iterating matrix to deflate. A similar phenomenon was observed by Wilkinson in the symmetric tridiagonal $Q R$ with the Rayleigh shift [12, p.414].

In this paper a further improvement is made over the shift strategy to eliminate the above-mentioned drawback. Based on the fact that eigenvalues of unitary matrices are all located on the unit circle, we modify the Wilkinson shift by projecting an appropriate Schur parameter of the iterating unitary Hessenberg matrix onto the unit circle so that the shift chosen (as an eigenvalue of the modified lower right 2-by-2 submatrix) is always unimodular 1 (In the conventional form, the shift is situated inside the circle and, in extreme cases, could be fairly small or even

Received by the editor May 5, 1999 and, in revised form, April 3, 2001.

2000 Mathematics Subject Classification. Primary 65F15, $15 \mathrm{~A} 18$.

Key words and phrases. QR algorithm, shift strategy, unitary Hessenberg matrices.

The first author's research was supported by the Center for Computational Sciences at the University of Kentucky.

The second author's research was supported in part by the National Science Foundation under grant DMS-8704196.

${ }^{1}$ With unimodular shifts, a root-free version of the unitary $Q R$ algorithm has been devised 10 in which the number of arithmetic operations is reduced by about 25 percent from that given in [2].

(C)2002 American Mathematical Society 
zero.) The idea of using unimodular shifts in the unitary case is quite natural, but the hard part is the mathematical proof of global convergence with this modified Wilkinson shift. Use is made of the fact that, on the unit circle, the modulus form of the Szegö recurrence relations for a unitary matrix is simpler to deal with. The main results are, with the unimodular Wilkinson shift, the $Q R$ iteration converges globally and the asymptotic rate is at least quadratic 7]; experimental testing also indicates that the modified shift gives more rapid numerical convergence than its traditional counterpart, especially in those extreme cases.

\section{NOTATION AND BACKGROUND}

Throughout, $U \in \mathbf{C}^{n \times n}$ will represent a unitary Hessenberg matrix with positive elements $\beta_{k}:=e_{k+1}^{*} U e_{k}, 1 \leq k<n$, on the subdiagonal and zero entries below it, where $e_{k}$ denotes the $k$ th column of the identity matrix and $e_{k}^{*}$ the conjugate transpose of $e_{k}$. The same structure and similar symbols for the subdiagonal elements apply to $\hat{U}$ and $U^{(k)}$, which are defined later. In general, small Greek letters are reserved for scalars. The set of the eigenvalues of $U$ is denoted by $\lambda(U)$, and the order of $U$ is assumed to be at least 3 .

2.1. The unitary $Q R$ algorithm. Given a unitary Hessenberg matrix $U$ and a nonzero shift $\lambda \in \mathbf{C}$, consider the unitary-triangular factorization of

$$
U-\lambda I=: Q R,
$$

where $Q$ is unitary and $R$ is upper triangular with nonnegative diagonal elements. This factorization is the matrix formulation of the Gram-Schmidt orthonormalizing process applied to the columns of $U-\lambda I$ from left to right, and hence $Q$ is also Hessenberg. Let $\sigma_{k}:=e_{k+1}^{*} Q e_{k}, 1 \leq k<n$, be the subdiagonal elements of $Q$ and let $\rho_{k}:=e_{k}^{*} R e_{k}, 1 \leq k \leq n$, be the diagonal elements of $R$. Note that the factorization is unique if $\lambda \notin \lambda(U)$. From $Q$ we define $\hat{U}$, the $Q R$ transform of $U$, by $\hat{U}:=Q^{*} U Q$. It is easy to check that

$$
\hat{U}-\lambda I=R Q,
$$

and that $\hat{U}$ is also (unitary) Hessenberg. We assume, with no loss of generality in theoretical analysis, that all the subdiagonal elements $\left\{\beta_{k}\right\}_{k=1}^{n-1}$ of $U$ are positive; consequently, with $\lambda \notin \lambda(U)$ and $\left\{\rho_{k}\right\}_{k=1}^{n}$ being positive, all the subdiagonal elements $\left\{\sigma_{k}\right\}_{k=1}^{n-1}$ of $Q$ and $\left\{\hat{\beta}_{k}\right\}_{k=1}^{n-1}$ of $\hat{U}$ are also positive for each $Q R$ step $U \rightarrow \hat{U}$. This is readily seen by equating the corresponding subdiagonal elements on each side of the matrix equations (2.1) and (2.2), respectively:

$$
\begin{aligned}
& \beta_{k}=\sigma_{k} \rho_{k}, \\
& \hat{\beta}_{k}=\sigma_{k} \rho_{k+1}, \quad 1 \leq k<n .
\end{aligned}
$$

Observe that

$$
\lambda \in \lambda(U) \Longleftrightarrow \rho_{n}=0 \Longleftrightarrow \hat{\beta}_{n-1}=0 \Longrightarrow \hat{\alpha}_{n n}:=e_{n}^{*} \hat{U} e_{n}=\lambda .
$$


The unitary $Q R$ algorithm [2] iterates the $Q R$ transformation $U \rightarrow \hat{U}$, with an appropriate nonzero shift $\lambda$ selected at each step:

$$
\begin{gathered}
U^{(1)}:=U, \\
\text { for } k=1,2,3, \ldots \\
U^{(k)}-\lambda^{(k)} I=: Q^{(k)} R^{(k)}, \\
U^{(k+1)}:=R^{(k)} Q^{(k)}+\lambda^{(k)} I,
\end{gathered}
$$

and a sequence of unitarily similar Hessenberg matrices $U^{(k)}$ (unreduced if $\lambda^{(k)} \notin$ $\lambda(U))$ is produced. The remarkable fact is that, with the shift strategy suitably devised, $\beta_{n-1}^{(k)} \rightarrow 0$ rapidly as $k \rightarrow \infty$ [12, [1], [4, 9]. Numerically, as $\beta_{n-1}^{(k)}$ becomes negligible to working accuracy, $\alpha_{n n}^{(k)}:=e_{n}^{*} U^{(k)} e_{n}$ can be accepted as an (approximate) eigenvalue of $U$ and the computation continues with the submatrix obtained by deleting the last row and column; sequentially all the eigenvalues are computed and come out in turn [11, 6], [8].

2.2. Schur parameterization. Every unitary Hessenberg matrix $U$ with positive subdiagonal elements $\left\{\beta_{k}\right\}_{k=1}^{n-1}$ can be uniquely represented in the Schur parametric form $U\left(\alpha_{1}, \alpha_{2}, \ldots, \alpha_{n}\right)$ [2], 9]:

$$
U=\left[\begin{array}{ccccc}
-\bar{\alpha}_{0} \alpha_{1} & -\bar{\alpha}_{0} \beta_{1} \alpha_{2} & \cdots & -\bar{\alpha}_{0} \beta_{1} \beta_{2} \cdots \beta_{n-2} \alpha_{n-1} & -\bar{\alpha}_{0} \beta_{1} \beta_{2} \cdots \beta_{n-1} \alpha_{n} \\
\beta_{1} & -\bar{\alpha}_{1} \alpha_{2} & \cdots & -\bar{\alpha}_{1} \beta_{2} \cdots \beta_{n-2} \alpha_{n-1} & -\bar{\alpha}_{1} \beta_{2} \cdots \beta_{n-1} \alpha_{n} \\
& \beta_{2} & \ddots & \vdots & \vdots \\
& & \ddots & -\bar{\alpha}_{n-2} \alpha_{n-1} & -\bar{\alpha}_{n-2} \beta_{n-1} \alpha_{n} \\
& & & \beta_{n-1} & -\bar{\alpha}_{n-1} \alpha_{n}
\end{array}\right]
$$

where $\alpha_{1}, \alpha_{2}, \ldots, \alpha_{n}$ are called the Schur parameters of $U, \alpha_{0}:=1,\left|\alpha_{n}\right|=1$,

$$
\left|\alpha_{k}\right|^{2}+\beta_{k}^{2}=1, \quad \alpha_{k} \in \mathbf{C}, \beta_{k}>0, \quad 1 \leq k<n,
$$

and

$$
\alpha_{j k}:=e_{j}^{*} U e_{k}=-\bar{\alpha}_{j-1} \beta_{j} \beta_{j+1} \cdots \beta_{k-1} \alpha_{k}, \quad 1 \leq j \leq k \leq n .
$$

Similarly, the matrix $Q$ in the $Q R$ factorization of $U-\lambda I$ is also unitary Hessenberg with positive subdiagonal elements $\left\{\sigma_{k}\right\}_{k=1}^{n-1}$ and, accordingly, can also be represented in the Schur form $Q\left(\gamma_{1}, \gamma_{2}, \ldots, \gamma_{n}\right)$ as $(2.5)$ with parameters $\gamma_{0}:=1$, $\left|\gamma_{n}\right|=1$, and

$$
\left|\gamma_{k}\right|^{2}+\sigma_{k}^{2}=1, \quad \gamma_{k} \in \mathbf{C}, \sigma_{k}>0, \quad 1 \leq k<n
$$

2.3. A unimodular Wilkinson shift. Based upon the fact that the eigenvalues of $U$ are all situated on the unit circle, we invariably use shifts of unit magnitude throughout the $Q R$ iteration, and we modify the conventional definition of the Wilkinson shift by projecting the Schur parameter $\alpha_{n-2}$, which appears in the lower right 2-by-2 submatrix of $U$ (see (2.5)), onto the unit circle so that the shift $\lambda$ (hereafter briefly called the $\overline{\mathrm{W}}$-shift), taken as one of the eigenvalues of the projected unitary submatrix, is always of unit modulus $(|\lambda|=1)$. That is, the $\overline{\mathrm{W}}$-shift is chosen as that eigenvalue of

$$
\left[\begin{array}{cc}
-\frac{\bar{\alpha}_{n-2}}{\left|\alpha_{n-2}\right|} \alpha_{n-1} & -\frac{\bar{\alpha}_{n-2}}{\left|\alpha_{n-2}\right|} \beta_{n-1} \alpha_{n} \\
\beta_{n-1} & -\bar{\alpha}_{n-1} \alpha_{n}
\end{array}\right]
$$


which is closer to $-\bar{\alpha}_{n-1} \alpha_{n}$, if $\alpha_{n-2} \neq 0$; in case $\alpha_{n-2}=0$, replace the unimodular factor $\frac{\alpha_{n-2}}{\left|\alpha_{n-2}\right|}$ in (2.8) by $\alpha_{n}$. In terms of the Schur parameters of $U$, the $\overline{\mathrm{W}}$-shift $\lambda$ satisfies the following characteristic relations: If $\alpha_{n-2} \neq 0$, then

$$
\begin{gathered}
\lambda^{2}+\left(\frac{\bar{\alpha}_{n-2}}{\left|\alpha_{n-2}\right|} \alpha_{n-1}+\bar{\alpha}_{n-1} \alpha_{n}\right) \lambda+\frac{\bar{\alpha}_{n-2}}{\left|\alpha_{n-2}\right|} \alpha_{n}=0, \\
\left|\bar{\alpha}_{n-1} \alpha_{n}+\lambda\right| \leq \beta_{n-1} \leq\left|\frac{\bar{\alpha}_{n-2}}{\left|\alpha_{n-2}\right|} \alpha_{n-1}+\lambda\right|
\end{gathered}
$$

if $\alpha_{n-2}=0$, replace $\frac{\alpha_{n-2}}{\left|\alpha_{n-2}\right|}$ in the above two relations by $\alpha_{n}$.

Remark. In the extreme case $\alpha_{n-2}=0$, it does not matter how we define the $\overline{\mathrm{W}}$ shift $\lambda$, as long as the conditions $|\lambda|=1$ and $\left|\bar{\alpha}_{n-1} \alpha_{n}+\lambda\right| \leq \beta_{n-1}$ (the lower bound in (2.10)) are satisfied; this is made clear later in the proof of Lemma 4(a) in Section 3.

2.4. Szegö recurrence relations. Consider the factorization $U-\lambda I=Q R$. Let $\left\{\chi_{k}\right\}_{k=1}^{n}$ be the characteristic polynomials of the successive leading principal submatrices $\left\{U_{k}\right\}_{k=1}^{n}$ of $U$. Then, with $Q$ in its Schur parametric form $Q\left(\gamma_{1}, \gamma_{2}, \ldots, \gamma_{n}\right)$, each $\chi_{k}$ can be expressed as a product of the parameter $\gamma_{k}$ and the diagonal elements $\left\{\rho_{j}\right\}_{j=1}^{k}$ of $R$ (for details, see [9]):

$$
\chi_{k}=\chi_{k}(\lambda):=\operatorname{det}\left(\lambda I_{k}-U_{k}\right)=\rho_{1} \rho_{2} \cdots \rho_{k} \gamma_{k}, \quad 1 \leq k \leq n .
$$

These polynomials are usually called the (monic) Szegö polynomials associated with $U\left(\alpha_{1}, \alpha_{2}, \ldots, \alpha_{n}\right)$, and they satisfy the Szegö recurrence relations [2], 9]:

$$
\begin{aligned}
& \chi_{0}:=1, \quad \tilde{\chi}_{0}:=1 \\
& \chi_{k}=\lambda \chi_{k-1}+\alpha_{k} \tilde{\chi}_{k-1}, \\
& \tilde{\chi}_{k}=\tilde{\chi}_{k-1}+\bar{\alpha}_{k} \lambda \chi_{k-1}, \quad 1 \leq k \leq n,
\end{aligned}
$$

where the auxiliary polynomials $\tilde{\chi}_{k}$ satisfy the relation $\tilde{\chi}_{k}(\lambda)=\lambda^{k} \chi_{k}^{c}(1 / \lambda)$, with the superscript $c$ denoting conjugation of the coefficients of a polynomial. We may also put $\tilde{\chi}_{k}$ in product form similar to that in $(2.11)$ for $\chi_{k}$ :

$$
\tilde{\chi}_{k}=: \rho_{1} \rho_{2} \cdots \rho_{k} \tilde{\gamma}_{k}, \quad 1 \leq k \leq n .
$$

Then, since $\rho_{1} \rho_{2} \cdots \rho_{n-1}>0,(2.12)$ can be replaced by

$$
\begin{aligned}
& \gamma_{0}:=1, \quad \tilde{\gamma}_{0}:=1 \\
& \rho_{k} \gamma_{k}=\lambda \gamma_{k-1}+\alpha_{k} \tilde{\gamma}_{k-1}, \\
& \rho_{k} \tilde{\gamma}_{k}=\tilde{\gamma}_{k-1}+\bar{\alpha}_{k} \lambda \gamma_{k-1}, \quad 1 \leq k \leq n .
\end{aligned}
$$

We have, as a special case of the Christoffel-Darboux-Szegö formula [3, p.192],

$$
\left|\tilde{\gamma}_{k}\right|^{2}+|\lambda|^{2} \sigma_{k}^{2}=1, \quad 1 \leq k \leq n,
$$

where $\sigma_{n}:=0$. (See 9] for a simple proof of this identity.) Note that for $|\lambda|=1$ we obtain, from $(2.7),(2.15),(2.11)$, and (2.13),

$$
\left|\gamma_{k}\right|=\left|\tilde{\gamma}_{k}\right| \text { and }\left|\chi_{k}\right|=\left|\tilde{\chi}_{k}\right|, \quad 1 \leq k \leq n .
$$




\section{Convergence of the $Q R$ iteration}

3.1. Properties related to convergence. We say the $Q R$ iteration converges if the last subdiagonal element $\beta_{n-1}^{(k)}$ of $U^{(k)}$ converges to zero. The following theorem about convergence is analogous to Theorem 6 of 9 with the conventional shifts for normal matrices, and the proof is therefore omitted. Here, we use instead a projected unimodular shift for unitary matrices in the iteration.

Theorem 1 (Properties of convergence). Let $U^{(k)}$ be the $Q R$ iterates of $U$ with the $\overline{\mathrm{W}}$-shift $\lambda^{(k)}$. If $\beta_{n-1}^{(k)} \rightarrow 0$ as $k \rightarrow \infty$, then

(a) $\lambda^{(k)} \rightarrow \lambda_{n}$ for some $\lambda_{n} \in \lambda(U)$,

(b) $\left|\chi_{n-1}^{(k)}\left(\lambda^{(k)}\right)\right| \geq \delta^{n-1}+O(\varepsilon)$, where $\delta:=\min _{j \neq k}\left\{\left|\lambda_{j}-\lambda_{k}\right|: \lambda_{j}, \lambda_{k} \in \lambda(U)\right\}>$ 0 and $\varepsilon$ is an arbitrarily small number,

(c) $\rho_{n}^{(k)} \rightarrow 0$, and $\left\{\rho_{j}^{(k)}\right\}_{j=1}^{n-1}$ are bounded away from zero.

To estimate the order of convergence of $\beta_{n-1}^{(k)}$ to zero, a relation between $\beta_{n-1}$ and $\hat{\beta}_{n-1}$ is needed for one $Q R$ step. Jiang and Zhang [4, Lemma 2] gave a relation for real symmetric tridiagonal matrices. A similar relation has been extended to (upper) Hessenberg matrices in [9, Lemma 4], which is given below:

Rate estimate. For each $Q R$ transformation $U \rightarrow \hat{U}$ (unitariness is not a requirement),

$$
\hat{\beta}_{n-1}=\left[\frac{\rho_{1} \rho_{2} \cdots \rho_{n-2}\left|\chi_{n}(\lambda)\right|}{\left(\rho_{1} \rho_{2} \cdots \rho_{n-2} \beta_{n-1}\right)^{2}+\left|\chi_{n-1}(\lambda)\right|^{2}}\right] \beta_{n-1} .
$$

This relation is indeed an identity, and can be easily checked through the use of (2.3), (2.4), (2.11), and (2.7).

3.2. A fundamental upper bound. The Schur parameters $\left\{\alpha_{k}\right\}_{k=1}^{n},\left\{\gamma_{k}\right\}_{k=1}^{n}$ of $U$ and $Q$, plus the diagonal elements $\left\{\rho_{k}\right\}_{k=1}^{n}$ of $R$, are interrelated through the equation $U-\lambda I=Q R$. With a unimodular shift $|\lambda|=1$, inequalities involved with the recurrence relations (2.14) can be greatly simplified by exploiting the equalmodulus relation (2.16). In the following lemma a least upper bound for $\sigma_{k}$ can be expressed in terms of $\beta_{k}$ and $\sigma_{k-1}$ [7]. This bound is fundamental to the proof of global convergence of $Q R$ and will be used repeatedly in the subsequent section. To simplify the mathematical expressions in our analysis, we use the notational convention

$$
\left\langle\beta_{k}\right\rangle:=\left(\frac{\beta_{k}}{1+\sqrt{1-\beta_{k}^{2}}}\right)^{2}, \quad 1 \leq k<n
$$

Then clearly

$$
0<\left\langle\beta_{k}\right\rangle \leq \beta_{k} \leq 1
$$

and

$$
\begin{aligned}
& \beta_{k} \rightarrow 0 \Longleftrightarrow\left\langle\beta_{k}\right\rangle \rightarrow 0, \\
& \beta_{k} \rightarrow 1 \Longleftrightarrow\left\langle\beta_{k}\right\rangle \rightarrow 1 .
\end{aligned}
$$

Note the one-to-one correspondence between $\beta_{k}$ and $\left\langle\beta_{k}\right\rangle$. 
Lemma 2. Let $U-\lambda I=Q R$ with $|\lambda|=1$. Then, for $1 \leq k<n$,

$$
0<\frac{1}{\sqrt{1+\left\langle\beta_{k}\right\rangle^{-1}\left(1-\sigma_{k-1}^{2}\right)}} \leq \sigma_{k} \leq \frac{1}{\sqrt{1+\left\langle\beta_{k}\right\rangle\left(1-\sigma_{k-1}^{2}\right)}}<1 .
$$

Proof. Applying the triangle inequality to the recurrence relations in (2.14), using (2.16), and squaring both sides of the inequality, we obtain

$$
\rho_{k}^{2}\left|\gamma_{k}\right|^{2} \geq\left(1-\left|\alpha_{k}\right|\right)^{2}\left|\gamma_{k-1}\right|^{2}
$$

which is equivalent to

$$
\frac{1}{\sigma_{k}^{2}} \geq 1+\left(\frac{\beta_{k}}{1+\sqrt{1-\beta_{k}^{2}}}\right)^{2}\left(1-\sigma_{k-1}^{2}\right),
$$

using the fact that $\rho_{k}^{2}=\beta_{k}^{2} / \sigma_{k}^{2},\left|\gamma_{k}\right|^{2}=1-\sigma_{k}^{2}$, and $1-\left|\alpha_{k}\right|=\beta_{k}^{2} /\left(1+\sqrt{1-\beta_{k}^{2}}\right)$ (cf. (2.3), (2.7), and (2.6)). Therefore,

$$
\sigma_{k} \leq \frac{1}{\sqrt{1+\left(\frac{\beta_{k}}{1+\sqrt{1-\beta_{k}^{2}}}\right)^{2}\left(1-\sigma_{k-1}^{2}\right)}}=: \frac{1}{\sqrt{1+\left\langle\beta_{k}\right\rangle\left(1-\sigma_{k-1}^{2}\right)}} .
$$

Since $\gamma_{0}:=1, \sigma_{0}:=0$ and $0<\beta_{k} \leq 1$, the right-hand side of the inequality is strictly less than unity. The lower bound on $\sigma_{k}$ follows similarly by applying the triangle inequality to (2.14) the other way and squaring:

$$
\rho_{k}^{2}\left|\gamma_{k}\right|^{2} \leq\left(1+\left|\alpha_{k}\right|\right)^{2}\left|\gamma_{k-1}\right|^{2} .
$$

3.3. Convergence with the unimodular shift. The proof of global convergence is based on the monotonic decrease of $\beta_{n-2}^{(k)} \beta_{n-1}^{(k)}$ and its connection with $\beta_{n-1}^{(k+1) 2}$ 7]. To establish these relations (in Lemma 4), we begin with a preparatory lemma.

Lemma 3. Let $U-\lambda I=Q R$ with $|\lambda|=1$. Then

(a) $\sqrt{1-\sigma_{n-3}^{2}} \sigma_{n-2} \sigma_{n-1}^{2}<\frac{\sqrt{1+\left\langle\beta_{n-2}\right\rangle}}{1+\left\langle\beta_{n-2}\right\rangle+\left\langle\beta_{n-2}\right\rangle\left\langle\beta_{n-1}\right\rangle}$,

(b) (i) $\left(1+\left\langle\beta_{n-2}\right\rangle\right) \sqrt{1-\sigma_{n-3}^{2}} \sigma_{n-2}^{2} \sigma_{n-1} \leq \omega\left(\left\langle\beta_{n-2}\right\rangle,\left\langle\beta_{n-1}\right\rangle\right)<1$,

(ii) $\omega\left(\left\langle\beta_{n-2}\right\rangle,\left\langle\beta_{n-1}\right\rangle\right) \rightarrow 1 \Longrightarrow\left\langle\beta_{n-2}\right\rangle\left\langle\beta_{n-1}\right\rangle \rightarrow 0 \Longleftrightarrow \beta_{n-2} \beta_{n-1} \rightarrow 0$, where the defining formula and basic properties of $\omega\left(\left\langle\beta_{n-2}\right\rangle,\left\langle\beta_{n-1}\right\rangle\right)$ are given in the Appendix.

Proof. (a) From Lemma 2 we have

$$
1-\sigma_{n-2}^{2} \geq 1-\frac{1}{1+\left\langle\beta_{n-2}\right\rangle\left(1-\sigma_{n-3}^{2}\right)}=\frac{\left\langle\beta_{n-2}\right\rangle\left(1-\sigma_{n-3}^{2}\right)}{1+\left\langle\beta_{n-2}\right\rangle\left(1-\sigma_{n-3}^{2}\right)}
$$

and

$$
\begin{aligned}
\sigma_{n-1}^{2} & \leq \frac{1}{1+\left\langle\beta_{n-1}\right\rangle\left(1-\sigma_{n-2}^{2}\right)} \leq \frac{1}{1+\left\langle\beta_{n-1}\right\rangle\left[\frac{\left\langle\beta_{n-2}\right\rangle\left(1-\sigma_{n-3}^{2}\right)}{1+\left\langle\beta_{n-2}\right\rangle\left(1-\sigma_{n-3}^{2}\right)}\right]} \\
& =\frac{1+\left\langle\beta_{n-2}\right\rangle\left(1-\sigma_{n-3}^{2}\right)}{1+\left\langle\beta_{n-2}\right\rangle\left(1+\left\langle\beta_{n-1}\right\rangle\right)\left(1-\sigma_{n-3}^{2}\right)} .
\end{aligned}
$$


Therefore,

$$
\begin{aligned}
& \sqrt{1-\sigma_{n-3}^{2}} \sigma_{n-2} \sigma_{n-1}^{2} \\
\leq & \sqrt{\frac{\left(1-\sigma_{n-3}^{2}\right)}{1+\left\langle\beta_{n-2}\right\rangle\left(1-\sigma_{n-3}^{2}\right)}}\left[\frac{1+\left\langle\beta_{n-2}\right\rangle\left(1-\sigma_{n-3}^{2}\right)}{1+\left\langle\beta_{n-2}\right\rangle\left(1+\left\langle\beta_{n-1}\right\rangle\right)\left(1-\sigma_{n-3}^{2}\right)}\right],
\end{aligned}
$$

from (3.4) and (3.5),

$$
\begin{aligned}
& =\frac{\sqrt{\left(1-\sigma_{n-3}^{2}\right)\left[1+\left\langle\beta_{n-2}\right\rangle\left(1-\sigma_{n-3}^{2}\right)\right]}}{1+\left\langle\beta_{n-2}\right\rangle\left(1+\left\langle\beta_{n-1}\right\rangle\right)\left(1-\sigma_{n-3}^{2}\right)} \\
& <\frac{\sqrt{1+\left\langle\beta_{n-2}\right\rangle}}{1+\left\langle\beta_{n-2}\right\rangle+\left\langle\beta_{n-2}\right\rangle\left\langle\beta_{n-1}\right\rangle},
\end{aligned}
$$

by a simple derivative calculation using the fact that $0<1-\sigma_{n-3}^{2}<1$.

(b) Again from (3.4) and (3.5) it follows that

$$
\begin{aligned}
& \left(1+\left\langle\beta_{n-2}\right\rangle\right) \sqrt{1-\sigma_{n-3}^{2}} \sigma_{n-2}^{2} \sigma_{n-1} \\
\leq & \left(1+\left\langle\beta_{n-2}\right\rangle\right) \sqrt{\frac{\left(1-\sigma_{n-3}^{2}\right)}{\left[1+\left\langle\beta_{n-2}\right\rangle\left(1-\sigma_{n-3}^{2}\right)\right]\left[1+\left\langle\beta_{n-2}\right\rangle\left(1+\left\langle\beta_{n-1}\right\rangle\right)\left(1-\sigma_{n-3}^{2}\right)\right]}} .
\end{aligned}
$$

For each $0<\left\langle\beta_{n-2}\right\rangle \leq 1,0<\left\langle\beta_{n-1}\right\rangle \leq 1$, the above expression, treated as a function of $\left(1-\sigma_{n-3}^{2}\right)$, has a least upper bound $\omega\left(\left\langle\beta_{n-2}\right\rangle,\left\langle\beta_{n-1}\right\rangle\right)$ which, together with the properties stated in (i) and (ii), is derived in the Appendix. Note that $\left\langle\beta_{n-2}\right\rangle\left\langle\beta_{n-1}\right\rangle \rightarrow 0 \Longleftrightarrow \beta_{n-2} \beta_{n-1} \rightarrow 0$ follows directly from (3.2).

Lemma 4. Let $\hat{U}$ be the $Q R$ transform of $U$ with the $\overline{\mathrm{W}}$-shift $\lambda$. Then

(a) $\left|\chi_{n}(\lambda)\right| \leq\left(1+\left\langle\beta_{n-2}\right\rangle\right) \beta_{n-2}^{2} \beta_{n-1}\left|\chi_{n-3}(\lambda)\right|$,

(b) $\hat{\beta}_{n-1}<\left[\frac{\left(1+\left\langle\beta_{n-2}\right\rangle\right) \sqrt{1+\left\langle\beta_{n-2}\right\rangle}}{1+\left\langle\beta_{n-2}\right\rangle+\left\langle\beta_{n-2}\right\rangle\left\langle\beta_{n-1}\right\rangle}\right] \beta_{n-2}<\sqrt{2} \beta_{n-2}$,

(c) $\hat{\beta}_{n-2} \hat{\beta}_{n-1} \leq \omega\left(\left\langle\beta_{n-2}\right\rangle,\left\langle\beta_{n-1}\right\rangle\right) \beta_{n-2} \beta_{n-1}<\beta_{n-2} \beta_{n-1}$,

(d) $\hat{\beta}_{n-1} \leq \sqrt{2} \beta_{n-1}$.

Proof. With recurrence relations (2.12) $\chi_{n}$ can be expressed in terms of $\chi_{n-3}$ and $\tilde{\chi}_{n-3}$ :

$$
\begin{aligned}
\chi_{n}= & \lambda\left[\lambda^{2}+\left(\bar{\alpha}_{n-2} \alpha_{n-1}+\bar{\alpha}_{n-1} \alpha_{n}\right) \lambda+\bar{\alpha}_{n-2} \alpha_{n}\right] \chi_{n-3} \\
& +\left[\alpha_{n-2} \lambda^{2}+\left(\alpha_{n-2} \bar{\alpha}_{n-1} \alpha_{n}+\alpha_{n-1}\right) \lambda+\alpha_{n}\right] \tilde{\chi}_{n-3} .
\end{aligned}
$$

Assume $\alpha_{n-2} \neq 0$. Then, combining (3.6) with the characteristic equation (2.9) for the $\overline{\mathrm{W}}$-shift $\lambda$, we get

$$
\chi_{n}=\alpha_{n} \lambda\left(1-\left|\alpha_{n-2}\right|\right)\left(\alpha_{n-1} \bar{\alpha}_{n}+\bar{\lambda}\right)\left(-\frac{\bar{\alpha}_{n-2}}{\left|\alpha_{n-2}\right|} \lambda \chi_{n-3}+\tilde{\chi}_{n-3}\right) .
$$

Taking the modulus on each side and applying the triangle inequality to the last factor on the right, we obtain, on condition that $|\lambda|=1$,

$$
\begin{aligned}
\left|\chi_{n}\right| & \leq 2\left(1-\left|\alpha_{n-2}\right|\right)\left|\bar{\alpha}_{n-1} \alpha_{n}+\lambda\right|\left|\chi_{n-3}\right|, & & \text { by }(2.16), \\
& \leq 2\left(1-\left|\alpha_{n-2}\right|\right) \beta_{n-1}\left|\chi_{n-3}\right|, & & \text { from (2.10), } \\
& =\left(1+\left\langle\beta_{n-2}\right\rangle\right) \beta_{n-2}^{2} \beta_{n-1}\left|\chi_{n-3}\right|, & & \text { using (2.6) and (3.2). }
\end{aligned}
$$

If $\alpha_{n-2}=0$, then (3.6) reduces to

$$
\chi_{n}=\lambda^{2}\left(\lambda+\bar{\alpha}_{n-1} \alpha_{n}\right) \chi_{n-3}+\left(\alpha_{n-1} \lambda+\alpha_{n}\right) \tilde{\chi}_{n-3}
$$


and (a) still holds (with $\beta_{n-2}=\left\langle\beta_{n-2}\right\rangle=1$ ), because for $|\lambda|=1$,

$$
\left|\alpha_{n-1} \lambda+\alpha_{n}\right|=\left|\lambda+\bar{\alpha}_{n-1} \alpha_{n}\right| \leq \beta_{n-1} \text {, from (2.10), }
$$

and $\left|\chi_{n-3}\right|=\left|\tilde{\chi}_{n-3}\right|$. This completes (a).

Next, applying relations (2.11), (2.7) and (2.3) to the inequality in (a) we obtain

$$
\rho_{n} \leq\left(1+\left\langle\beta_{n-2}\right\rangle\right) \sqrt{1-\sigma_{n-3}^{2}} \sigma_{n-2} \sigma_{n-1} \beta_{n-2}
$$

and, equivalently,

$$
\rho_{n-1} \rho_{n} \leq\left(1+\left\langle\beta_{n-2}\right\rangle\right) \sqrt{1-\sigma_{n-3}^{2}} \sigma_{n-2} \beta_{n-2} \beta_{n-1} .
$$

Therefore we have

$$
\begin{aligned}
\hat{\beta}_{n-1} & =\sigma_{n-1} \rho_{n}, & & \text { by (2.4), } \\
& \leq\left[\left(1+\left\langle\beta_{n-2}\right\rangle\right) \sqrt{1-\sigma_{n-3}^{2}} \sigma_{n-2} \sigma_{n-1}^{2}\right] \beta_{n-2}, & & \text { from (3.7), } \\
& \left.<-\frac{\left(1+\left\langle\beta_{n-2}\right\rangle\right) \sqrt{1+\left\langle\beta_{n-2}\right\rangle}}{1+\left\langle\beta_{n-2}\right\rangle+\left\langle\beta_{n-2}\right\rangle\left\langle\beta_{n-1}\right\rangle}\right] \beta_{n-2}, & & \text { from Lemma 3(a), } \\
& <\sqrt{1+\left\langle\beta_{n-2}\right\rangle} \beta_{n-2} \leq \sqrt{2} \beta_{n-2}, & & \text { using (3.3), }
\end{aligned}
$$

and

$$
\begin{aligned}
\hat{\beta}_{n-2} & \hat{\beta}_{n-1} & & \\
& =\sigma_{n-2} \sigma_{n-1} \rho_{n-1} \rho_{n}, & & \text { by (2.4), } \\
& \leq\left(1+\left\langle\beta_{n-2}\right\rangle\right) \sqrt{1-\sigma_{n-3}^{2}} \sigma_{n-2}^{2} \sigma_{n-1} \beta_{n-2} \beta_{n-1}, & & \text { from (3.8), } \\
& \leq \omega\left(\left\langle\beta_{n-2}\right\rangle,\left\langle\beta_{n-1}\right\rangle\right) \beta_{n-2} \beta_{n-1}<\beta_{n-2} \beta_{n-1}, & & \text { from Lemma 3(b)(i). }
\end{aligned}
$$

These give (b) and (c), respectively.

Finally, to prove $(\mathrm{d})$, note that

$$
\begin{aligned}
\hat{\beta}_{n-1} & \leq \rho_{n} \\
& \leq\left\|R e_{n}\right\|=\left\|Q R e_{n}\right\|=\left\|(U-\lambda I) e_{n}\right\| \\
& =\left\|e_{n}^{*}(U-\lambda I)\right\|=\sqrt{\beta_{n-1}^{2}+\left|\bar{\alpha}_{n-1} \alpha_{n}+\lambda\right|^{2}} \\
& \leq \sqrt{2} \beta_{n-1},
\end{aligned}
$$

from the basic properties related to the $Q R$ transformation $U \rightarrow \hat{U}$ and (2.10), a characteristic inequality for the $\overline{\mathrm{W}}$-shift $\lambda$.

We now arrive at the major result of our theoretical analysis [7.

Theorem 5 (Global convergence). Let $U^{(k)}$ be the $Q R$ iterates of $U$ with the exclusive use of the $\overline{\mathrm{W}}$-shift. Then $\beta_{n-1}^{(k)} \rightarrow 0$.

Proof. From Lemma 4(c), we know that $\beta_{n-2}^{(k)} \beta_{n-1}^{(k)}$ form a bounded decreasing sequence which has a limit, say $\beta$. We claim $\beta=0$, because if $\beta>0$, then it would follow that $\omega\left(\left\langle\beta_{n-2}^{(k)}\right\rangle,\left\langle\beta_{n-1}^{(k)}\right\rangle\right) \rightarrow 1$ and, from Lemma 3(b)(ii), that $\beta_{n-2}^{(k)} \beta_{n-1}^{(k)} \rightarrow 0$, a contradiction. Hence $\beta_{n-1}^{(k)} \rightarrow 0$, because

$$
\beta_{n-1}^{(k+1) 2}<2 \beta_{n-2}^{(k)} \beta_{n-1}^{(k)} \rightarrow 0
$$

from (b) and (d) of Lemma 4. 
Unlike unitary $Q R$ with the Wilkinson shift (cubic order for $\beta_{n-1}^{(k)} \rightarrow 0$, as proved in [7, see also 9]), with the unimodular shift the order of convergence can only be shown to be quadratic.

Theorem 6 (Local convergence). Let $\hat{U}$ be the $Q R$ transform of $U$ with the $\overline{\mathrm{W}}$-shift $\lambda$. Then $\hat{\beta}_{n-1}=O\left(\beta_{n-2}^{2} \beta_{n-1}^{2}\right)$ as $\beta_{n-1} \rightarrow 0$.

Proof. The conclusion follows immediately from (3.1) and Lemma 4(a), since all the elements involved in the $Q R$ transformation are bounded and, as $\beta_{n-1} \rightarrow 0$, $\left|\chi_{n-1}(\lambda)\right|$ is bounded from below by Theorem 1(b).

\section{NUMERICAL EXPERIMENTS}

Two specific examples are given to demonstrate the superiority of the unimodular shift introduced in this paper. In each case the starting matrix $U=: U^{(1)}$ in the $Q R$ iteration is specified by assigning numerical values to its Schur parameters $\left\{\alpha_{j}\right\}_{j=1}^{n}$. The test was focused on determining the number of iterations required for $\beta_{m-1}^{(k)}$, the last subdiagonal element of the iterating Hessenberg matrix $U_{m}^{(k)}$ of size $m$, to become negligible at stages $m=n, n-1, \ldots, 2$; and the criterion we used is $\mathrm{fl}\left(1+\beta_{m-1}^{(k)}\right)=1$, that is, when $\beta_{m-1}^{(k)}$ is smaller than the unit roundoff $\varepsilon$. Computations were done in double-precision Fortran on an IBM compatible PC-80486 with $\varepsilon \approx 10^{-19}$.

In each experiment the results for $n=8$ are given. To facilitate the listing of data, the following contractions are used:

$$
\begin{aligned}
\text { EH } & \text { Eberlein-Huang shift (proposed in [1]), } \\
\mathrm{W}^{\prime} & \text { modified Wilkinson shift (described in [9]), } \\
\overline{\mathrm{W}} & \text { unimodular Wilkinson shift (proposed in Section 2.3), } \\
\text { itmax } & \text { maximum number of iterations required to get one eigenvalue, } \\
\text { itsum } & \text { total number of iterations required to get all the eigenvalues. }
\end{aligned}
$$

Experiment 1. For $1 \leq j \leq n-2, \alpha_{j}=1 / \sqrt{2} ; \alpha_{n-1}=10^{-7}, \alpha_{n}=1$.

\begin{tabular}{|l|lll|}
\hline$n=8$ & $\mathrm{EH}$ & $\mathrm{W}^{\prime}$ & $\overline{\mathrm{W}}$ \\
\hline itmax & 63 & 5 & 4 \\
itsum & 80 & 22 & 21 \\
\hline
\end{tabular}

Experiment 2. For $1 \leq j \leq n-2, \alpha_{j}=0 ; \alpha_{n-1}=10^{-7}, \alpha_{n}=\sqrt{-1}$.

\begin{tabular}{|l|lll|}
\hline$n=8$ & $\mathrm{EH}$ & $\mathrm{W}^{\prime}$ & $\overline{\mathrm{W}}$ \\
\hline itmax & 28 & 38 & 4 \\
itsum & 45 & 55 & 21 \\
\hline
\end{tabular}

Although the rate of $\beta_{n-1}^{(k)} \rightarrow 0$ is ultimately cubic for both $\mathrm{EH}$ and $\mathrm{W}^{\prime}$ [7, [9], with these shifts it may take numerous iterations for the (first) deflation to occur, as the above two examples demonstrate. This is mainly because, in the early stages of the iteration, the shift is too small (but not zero) to make a significant change of the iterating matrix from unitariness before the $Q R$ factorization. But eventually, as the magnitude of the shift keeps growing toward unity, an asymptotic regime is established and convergence is then very swift. In contrast, the $\overline{\mathrm{W}}$-shift produces very efficient numerical convergence in these examples. 
Large numbers of random unitary Hessenberg matrices were also tested for convergence with these shift strategies, and overall, the $\overline{\mathrm{W}}$-shift seems to be the choice for the unitary $Q R$, as the data in the following table illustrate. The numbers of iterations shown in the rows are the numerical averages over 3000 sets of "randomly selected" Schur parameters $\left\{\alpha_{j}\right\}_{j=1}^{n}$, and there are about $\pm 1 \%$ fluctuations in these averages when a different seed value is assigned to the random-number generator.

Experiment 3. Randomly selected $\left\{\alpha_{j}\right\}_{j=1}^{n}$ :

for $1 \leq j \leq n-1,0<\left|\alpha_{j}\right|<1 ;\left|\alpha_{n}\right|=1$.

\begin{tabular}{|l|lll|}
\hline$n=8$ & $\mathrm{EH}$ & $\mathrm{W}^{\prime}$ & $\overline{\mathrm{W}}$ \\
\hline itmax & 4.72 & 4.15 & 4.01 \\
itsum & 21.5 & 19.9 & 19.4 \\
\hline
\end{tabular}

\section{Appendix. BASIC PROperties of $\omega\left(\left\langle\beta_{n-2}\right\rangle,\left\langle\beta_{n-1}\right\rangle\right)$}

All the mathematical letter symbols used in this section represent positive real numbers less than or equal to one. Before we define $\omega\left(\left\langle\beta_{n-2}\right\rangle,\left\langle\beta_{n-1}\right\rangle\right)$, a lemma with simpler notation is presented.

Lemma. Let $f\left(x ; b_{1}, b_{2}\right):=\left(1+b_{2}\right) \sqrt{\frac{x}{\left(1+b_{2} x\right)\left(1+b_{2}\left(1+b_{1}\right) x\right)}}$, where $0<x<1,0<$ $b_{1} \leq 1,0<b_{2} \leq 1$. Then:

(a)

$$
g\left(b_{1}, b_{2}\right):=\sup _{0<x<1} f\left(x ; b_{1}, b_{2}\right)= \begin{cases}\sqrt{\frac{1+b_{2}}{1+b_{2}+b_{1} b_{2}}} & \text { if } b_{2} \leq \frac{1}{\sqrt{1+b_{1}}} \\ \frac{1+b_{2}}{\sqrt{b_{2}}\left(1+\sqrt{1+b_{1}}\right)} & \text { if } b_{2}>\frac{1}{\sqrt{1+b_{1}}}\end{cases}
$$

(b) $\quad 0<g\left(b_{1}, b_{2}\right)<1$; if $g\left(b_{1}, b_{2}\right) \rightarrow 1$, then $b_{1} b_{2} \rightarrow 0$.

Proof. (a) Take the derivative of $f\left(x ; b_{1}, b_{2}\right)$ with respect to $x$ and calculate.

(b) If $b_{2} \leq \frac{1}{\sqrt{1+b_{1}}}$, then

$$
g\left(b_{1}, b_{2}\right)=\sqrt{\frac{1+b_{2}}{1+b_{2}+b_{1} b_{2}}}<1 .
$$

If $b_{2}>\frac{1}{\sqrt{1+b_{1}}}$, then $\sqrt{1+b_{1}}>\frac{1}{b_{2}}$ and

$$
g\left(b_{1}, b_{2}\right)=\frac{1+b_{2}}{\sqrt{b_{2}}\left(1+\sqrt{1+b_{1}}\right)}<\frac{1+b_{2}}{\sqrt{b_{2}}\left(1+\frac{1}{b_{2}}\right)}=\sqrt{b_{2}} \leq 1 .
$$

Clearly, $0<g\left(b_{1}, b_{2}\right)<1$. If $g\left(b_{1}, b_{2}\right) \rightarrow 1$, then

$$
\left.\begin{array}{ll}
\text { in case (A.1) }: & b_{1} b_{2} \rightarrow 0 \\
\text { in case (A.2) }: & b_{2} \rightarrow 1 \text { and } b_{1} \rightarrow 0
\end{array}\right\} \Longrightarrow b_{1} b_{2} \rightarrow 0 .
$$

Setting $x:=1-\sigma_{n-3}^{2}, b_{1}:=\left\langle\beta_{n-1}\right\rangle$, and $b_{2}:=\left\langle\beta_{n-2}\right\rangle$ in the above lemma, we have

$$
\begin{gathered}
\left(1+\left\langle\beta_{n-2}\right\rangle\right) \sqrt{\frac{\left(1-\sigma_{n-3}^{2}\right)}{\left[1+\left\langle\beta_{n-2}\right\rangle\left(1-\sigma_{n-3}^{2}\right)\right]\left[1+\left\langle\beta_{n-2}\right\rangle\left(1+\left\langle\beta_{n-1}\right\rangle\right)\left(1-\sigma_{n-3}^{2}\right)\right]}} \\
=f\left(x ; b_{1}, b_{2}\right) \leq g\left(b_{1}, b_{2}\right)=: \omega\left(\left\langle\beta_{n-2}\right\rangle,\left\langle\beta_{n-1}\right\rangle\right),
\end{gathered}
$$

and this completes the proof of Lemma $3(\mathrm{~b})$. 


\section{ACKNOWLEDGMENTS}

The authors would like to thank the editor and the referee for providing helpful suggestions which led to improvements in the presentation of this paper. The work of the first author is based on part of his Ph.D. thesis [7]. A preliminary form of this research appeared as 10, which was supported by the Center for Computational Sciences at the University of Kentucky and in part by the National Science Foundation under grant DMS-8704196.

\section{REFERENCES}

1. P. J. Eberlein and C. P. Huang, Global convergence of the QR algorithm for unitary matrices with some results for normal matrices, SIAM J. Numer. Anal. 12 (1975), 97-104. MR 50:8948

2. W. B. Gragg, The $Q R$ algorithm for unitary Hessenberg matrices, J. Comput. Appl. Math. 16 (1986), 1-8.

3. W. B. Gragg, Positive definite Toeplitz matrices, the Arnoldi process for isometric operators, and Gaussian quadrature on the unit circle, J. Comput. Appl. Math. 46 (1993), 183-198. MR 94e:65046

4. E. Jiang and Z. Zhang, A new shift of the $Q L$ algorithm for irreducible symmetric tridiagonal matrices, Linear Algebra Appl. 65 (1985), 261-272. MR 86g:65082

5. R. S. Martin, G. Peters, and J. H. Wilkinson, The QR algorithm for real Hessenberg matrices, Numer. Math. 14 (1970), 219-231.

6. B. N. Parlett, The Symmetric Eigenvalue Problem, Prentice-Hall, Englewood Cliffs, NJ, 1980. MR 81j:65063

7. T.-L. Wang, Convergence of the $Q R$ algorithm with origin shifts for real symmetric tridiagonal and unitary Hessenberg matrices, Ph.D. thesis, University of Kentucky, Lexington, KY, 1988.

8. T.-L. Wang, Convergence of the tridiagonal QR algorithm, Linear Algebra Appl. 322 (2001), $1-17$.

9. T.-L. Wang and W. B. Gragg, Convergence of the shifted QR algorithm for unitary Hessenberg matrices, to appear in Math. Comp.

10. T.-L. Wang and W. B. Gragg, Convergence of the unitary Hessenberg QR algorithm with unimodular shifts, Report NPS-53-90-008, Naval Postgraduate School, Monterey, CA, 1990.

11. J. H. Wilkinson, The Algebraic Eigenvalue Problem, Clarendon Press, Oxford, 1965. MR 32:1894

12. J. H. Wilkinson, Global convergence of tridiagonal $Q R$ algorithm with origin shifts, Linear Algebra Appl. 1 (1968), 409-420. MR 38:2938

Department of Mathematical Sciences, National Chengchi University, Taipei, TaiWAN, RePUBlic OF ChinA

E-mail address: wang@math.nccu.edu.tw

Department of Mathematics, Naval Postgraduate School, Monterey, California 93943

E-mail address: gragg@nps.navy.mil 